\title{
Integration of Supply Chain in IBS Construction
}

\author{
Ani Saifuza Abd Shukor, Faridah Muhammad Halil, \\ Mohammad Fadhil Mohammad, Rohana Mahbub \\ Centre of Studies for Quantity Surveying, Faculty of Architecture, Planning \& Surveying, \\ Universiti Teknologi MARA (UiTM), 40450 Shah Alam, Selangor, Malaysia \\ asaswan74@yahoo.com
}

\begin{abstract}
To ensure the success of IBS construction, the research will look at the integration means and factors that helped to integrate the IBS supply chains. Findings from semi-structured interviews with key IBS supply chain players revealed, human and behavioural factors, supply chain process and exchange flow, supply chain structure and collaboration and the working environment should be developed amongst the IBS supply chains. The findings contribute enabling factors to improve integration within IBS supply chains in the construction environment in Malaysia.
\end{abstract}

Keywords: Construction environment; Industralised Building System; integration and supply chain

eISSN: 2398-4279 @ 2016.: 2398-4279 @ 2016. The Authors. Published for AMER ABRA by e-International Publishing House, Ltd., UK.. This is an open access article under the CC BY-NC-ND license (http://creativecommons.org/licenses/by-nc-nd/4.0/). Peer-review under responsibility of AMER (Association of Malaysian Environment-Behaviour Researchers), ABRA (Association of Behavioural Researchers on Asians) and cEBs (Centre for Environment-Behaviour Studies), Faculty of Architecture, Planning \& Surveying, Universiti Teknologi MARA, Malaysia.

https://doi.org/10.21834/ajqol.v1i3.20 


\subsection{Introduction}

Current construction practices in the Malaysian construction industry play a critical role in the successful delivery of projects. While challenges often occur in the area of productivity and the delivery of work, the establishment of the Construction Industry Master Plan (CIMP) and Construction Industry Transformation Programme (CITP) has led to initiatives in the implementation of innovative approaches through Industrialised Building System (IBS) towards the sustainable environment. According to Isnin et al., (2012), sustainable environment highly demand a high-level of skills and technical techniques. Thus, IBS is a strategy for transforming the construction industry into an industrialised sector that deals with prefabricated components with highly technical skills and techniques.

There are numerous benefits to and drivers in IBS; however, it raises challenges that need to be overcome, mostly related to the supply chains (SC) and integration (Abd Shukor et al., 2009; CREAM, 2009). One of the most crucial problems in IBS is Lack of SC integration and togetherness. IBS requires a high-level skill, technical techniques, coordination and integration of SC (Mohammad et al., 2014), thus, the industry should change and move towards more integrated and innovative ways of working practices. To ensure the success of IBS projects, integration should be developed amongst the SC and the construction supply chains involved need to understand the means and dimension factors in improving integration in construction.

\subsection{Literature Review}

In the Malaysian construction industry, the importance of IBS was highlighted under Strategic Thrust 5 of CIMP and being reinforced in 2003-2010 (1st phase) and 2011-2015 IBS Roadmap and 2016-2020 CITP. However, even though the re-introduction of the IBS construction method has long been introduced and has promised to solve and improve the current construction process, IBS practices have still contributed to project delay and faces a difficult task in establishing the integration between parties (CIDB, 2010). Shamsudin et al., (2013) mentioned that the adoption of IBS also requires more fundamental changes to the conventional working practices and the relationship amongst the players due to industrialised construction. There was a strong belief that integration supply chains and industrialisation was the key to better building production and were critical for improving the performance of the construction industry with the goal of being more integrated. About this, the Malaysian Government has been persuaded to employ the innovative approach of IBS and integrated SC to steer the construction industry in the right direction (CIDB, 2007).

However, Lack of integration among relevant players in the design stage has resulted in the need for plan redesigning and additional costs incurred if IBS is adopted. Rashid (2009) argued that the possible mismatch between the design capabilities of the local engineering consultants and the local building product manufacturers will disrupt or create upheavals in the IBS project delivery. Thus, to improve integration, Helou and Caddy (2006) name human factors that look at the people. The people should show better adaptation and have the desire to change and 
good commitment to improvement. Moreover, there must be a higher level of trust and more focus on managing relationships. Organisation will require change and arrangement of the SC structure to ease communication and knowledge transfer. Meanwhile, information technology adaptation is dependent on the implementation and operation of IT interface to enhance the interaction.

Interaction is achieved based on the organisation's dimensions of information flow and exchange, knowledge, financial and contractual relationship. Handfield and Nichols (1999) developed three (3) principal dimensions of an integrated supply chain model, which consists of flows of communication, information and inventory system and supply chain relationships. Similarly, Briscoe and Dainty (2005) suggested major means to be emphasised: i) managing the flow and exchange through developing effective communication system throughout the tiers, ii) integrating the system and the process with in-depth knowledge of the systems in order to add value and iii) considering the coordinated and collaboration needs in order to deliver better value to the client through embracing a partnering technique. Numerous studies also considered the importance of the notion of supply chain relationship aspects that highlight and sought the relationships between parties in the construction (Helou \& Caddy, 2006). Fernie et al. (1999) developed six (6) constructs of integration dimension with the addition of the attitude, culture and responsibility dimensions. Baiden (2006) revealed input factors on the effective integration of the perspectives of construction managers and postulated the same elements as above; however, the authors draw our attention to the a new element, which is the environmental factor that consists of the movement and interaction space of the players, location, linkage and the boundaries.

\subsection{Methodology}

The primary aim of this research is to identify the means and dimension factors influencing successful integration between IBS SC players. An extensive and in-depth review of the literature was done to obtain information on the related IBS construction, SC management and integration, project procurement delivery as well as SC means and dimension factors influencing the successful integration of SC issue in the construction. To further clarify the scenario of a more realistic expectation of an integrated IBS SC is presented by interviewing the members of supply chain separately to get a common view of how they will work together. The semi-structured interviews were used to gain in-depth knowledge of the concepts or variables being studied. This study was undertaken to gain insight and in-depth perspectives from the multiple participants on factors that lead to the success of SC integration.

\subsection{Administration and sampling}

The semi-structured interviews were face-to-face. All interviews were recorded and transcribed verbatim, with each interview lasted for 40 to 90 minutes. In this present research, the primary consideration in purposive sampling was the judgment of which people could provide the best 
information to achieve the research objectives since the reintroduction scene of IBS in the Malaysian construction industry leaves for a small sampling pool at this time. Thus, the research investigated the interaction of up to five different tiers of the SC, encompassing clients, designers, contractors, manufacturers/system providers and installers who deeply involved and experienced in the IBS approach. Surprisingly, within 35 IBS players selected, the number of the clients and the system providers/manufacturers respondents are limited. They are only four and five respondent respectively. The limited number of clients because of the clients they involved and handled one or more projects. That's the one who are knowledgeable in IBS and involved interaction between IBS supply chain in the IBS construction project. The limited respondent of system providers/manufacturers is due to the reintroduction of IBS in the Malaysian construction industry; they are the only and competent companies big enough to handle the majority of IBS projects. In total, 35 semi-structured interviews were carried out. The majority of the respondents were from the top management level and very experienced. Thus, the data and views expressed by the respondents are noteworthy because their position enabled them to provide reliable information.

\subsection{Data analyses}

To establish the raw data and the meaning of the interviews, content analysis and cognitive mapping techniques are used for analysing qualitative data supported by the Nvivo software. In this research, according to the interview protocol and guide, after the interviews were transcribed, the transcript was imported, and the contents were coded in Nvivo 9 software, which allows the concepts or 'nodes' to be displayed accordingly and helps the researcher manage the data efficiently. Based on the conceptual content analysis, the coded concepts were analysed based on the quantification. The comparison analysis of the IBS supply chain achieved the objectives of the research to analyse the key means and dimension that influence the successful of integration between the IBS supply chain players.

\subsection{Results and Discussions}

The findings revealed eight significant criteria that should be undertaken the under four main means: human and behavioural, supply chain process and exchange flow, supply chain structure and collaboration and working environment. Figures 1,Figure 2 (a)-(b) and Figure 3 (a)-(c) illustrate the cognitive map and Nvivo tree nodes that reveal the themes driven by the analysis. 


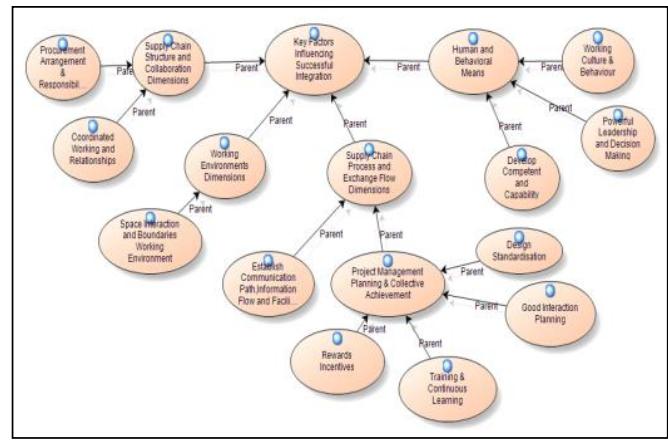

Figure 1: Cognitive mapping of the means and dimension influencing factors for successful improvement of integration

\begin{tabular}{|c|c|}
\hline \multicolumn{2}{|l|}{ Tree Nodes } \\
\hline * Name & D Sources \\
\hline Client Success & 0 \\
\hline -1. Human and Behavioral Dimensions & 0 \\
\hline Develop Competent and Capabilityr & 4 \\
\hline Powerful Leadership and Decision Making & 0 \\
\hline Working Culture \& Echaviour & 4 \\
\hline 10 Supply 'Chain Process and Exchange Flow Dimensions & 0 \\
\hline Establish Communication Path.Information Flow and Facilitate Proble & 3 \\
\hline 由. Project Management Planning \& Collective Achievement & 0 \\
\hline Design Standardisation & 1 \\
\hline Good Interaction Planning & 2 \\
\hline O Rewards Incentives & 0 \\
\hline . Training \& Continuous Learning & 1 \\
\hline 1. Supply Chain Structure and Collaboration Dimensions & 0 \\
\hline O Coordinated Working and Relationships & 3 \\
\hline Procurement Arrangement \& Responsibilities of SC & 4 \\
\hline W Working Environments Dimensions & 0 \\
\hline O Space Interaction and Bioundaries Working Environment & 0 \\
\hline
\end{tabular}

\begin{tabular}{|c|c|c|}
\hline \multicolumn{3}{|c|}{ Tree Nodes } \\
\hline 1 & Name & Sounces \\
\hline & Designer Success & 0 \\
\hline & Human and Behavioral Dimensions & 0 \\
\hline & Develop Competent and Capability & 11 \\
\hline & Powerful Leaders hip and Decision Making & 3 \\
\hline & Working Culture \& Behaviour & 11 \\
\hline & Supply Chain Process and Exchange Flow Dimensions & 0 \\
\hline & Establish Communication Path, Information Flow and Facilitate Proble & 9 \\
\hline & 1. Project Management Planning \& Collective Achievement & 0 \\
\hline & Design Standardisation & 5 \\
\hline & Good Interaction Planning & 6 \\
\hline & Rewards incentives & 0 \\
\hline & Training \& Continuous Learning & 4 \\
\hline & O Supply Chain Structure and Collaboration Dimensions & 0 \\
\hline & Coordinated Working and Relations hips & 9 \\
\hline & Procurement Arrangement \& Responsibilities of SC & 11 \\
\hline & 10 Working Environments Dimensions & 0 \\
\hline & O Space Interaction and Boundaries Working Environment & 0 \\
\hline
\end{tabular}

Figure 2: The means and dimension influencing factors for successful improvement of integration (a) group of clients (b) group of designers 


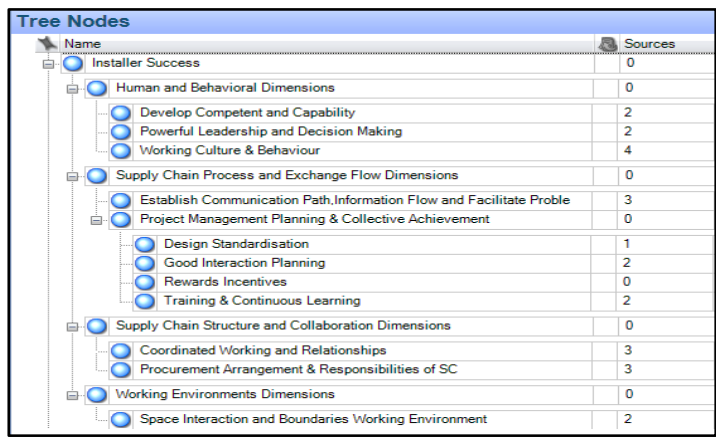

\begin{tabular}{|c|c|}
\hline \multicolumn{2}{|l|}{ Tree Nodes } \\
\hline 1 Name & Sources \\
\hline O Contractor Success & 0 \\
\hline 10 Human and Behavioral Dimensions & 0 \\
\hline Develop Competent and Capability & 9 \\
\hline Powerful Leaders hip and Decision Making & 4 \\
\hline Working Culture \& Behaviour & 7 \\
\hline 10 Supply Chain Process and Exchange Flow Dimensions & 0 \\
\hline Establish Communication Path. Information Flow and Facilitate Proble & 9 \\
\hline Project Management Planning \& Collective Achievement & 0 \\
\hline Design Standardisation & 3 \\
\hline Good Interaction Planning & 3 \\
\hline O. Rewards Incentives & 4 \\
\hline Training \& Continuous Learning & 2 \\
\hline 10 Supply Chain Structure and Collaboration Dimensions & 0 \\
\hline Coordinated Working and Relationships & 9 \\
\hline ... Procurement Arrangement \& Responsibilities of SC & 9 \\
\hline - Working Environuments Dimensions & 0 \\
\hline . Space Interaction and Bioundaries Working Enwironment & 2 \\
\hline
\end{tabular}

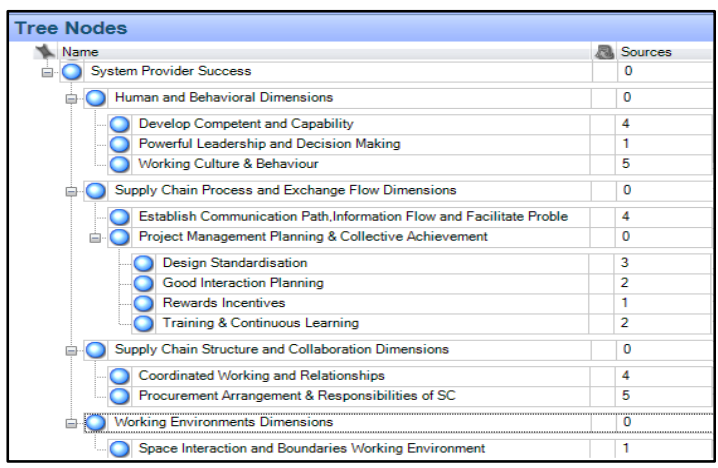

Figure 3: The means and dimension influencing factors for successful improvement of integration (a) group of main contractors (b) group of installers (c) group of system providers/manufacturers 


\subsubsection{Develop competent and capability}

The majority of IBS SC identified competence and capability as factors for the success of SC integration. This was particularly emphasised by clients. The IBS supply chains also emphasised that the upper and lower tiers of IBS supply chains should work together to develop an understanding of the scope of the work and technical knowledge such as design, pre-casting, construction and installation of IBS components.

\subsubsection{Powerful leadership in decision-making}

The results revealed that leadership in decision-making was identified as an important factor by designers, contractors, system providers/manufacturers and installers. The majority of respondents emphasised the client's role in leadership and decision making, while designers noted the power of contractors in decision making and in coordinating their supply chains. In the literature, many scholars have highlighted the importance of strong leadership, especially for clients, to drive greater integration

\subsection{SC process and exchange flow mean}

SC process and exchange flow include four factors: (1) establish a communication path, information exchange and facilitate problems (3) project management planning and collective achievements.

\subsubsection{Establish communication path, information exchange and facilitate problems}

In an integrated SC, communication plays an important role in how people handle, collect and transfer information. The clients, designers, contractors, system providers/manufacturers and installers suggested that to improve SC integration, IBS should consider using workshop sessions, briefings and progress meetings to establish communication and information flow. Furthermore, the issue of early involvement of system providers was also raised to discussing and sharing information at the early stages of a project. Also, designers noted the importance of early involvement for installers. Clients, designers, contractors and system providers/manufacturers also mentioned the importance of undertaking problem solving using win-win situations. Previous studies have noted the importance of brainstorming, joint discussions, frequent meetings and project workshops for promoting communication and information transfers (Briscoe \& Dainty, 2005).

\subsubsection{Project management planning and collective achievement}

Project management planning and collective achievements refer specifically to (1) project planning and execution, (2) design standardisation and modularisation, (3) training and continuous learning and (4) rewards incentive. The importance of training and continuous learning was noted by all players in the IBS SC. Players in the IBS SC noted that project management planning and execution by IBS contractors was one of the main factors in the successful integration. It was suggested that the IBS contractor should have a good and competent project manager on site (Jabar et al., 2013). All players in the SC mentioned that 
clients and designers should strive for effective modularisation and standardisation of concepts, where the priorities of the client are to emphasise standard design and modularisation and suitability for complex projects. Findings from the literature confirm the importance of technical planning and good project management for greater integration. Contractors and system providers/manufacturers raised the issue of collective achievements and the importance of rewards and incentives for celebrating milestones. This finding was previously reported in Hall (2001).

\subsection{SC structure and collaboration mean}

Two main factors in this category are (1) procurement arrangements and responsibilities of SC and (2) coordinated working and relationships.

\subsubsection{Procurement arrangement and responsibilities of SC}

The importance of procurement arrangements and SC responsibilities was emphasised by all IBS SC players. Clients, designers, contractors and system providers/manufacturers highlighted the need for contractors to assume responsibility and total control for Design and Build arrangements. Contractors, system providers/manufacturers and installers also emphasised that early involvement of system providers/manufacturers before the tender and design process are facilitated integration. All players in the SC reported that partnering and joint ventures enhanced integration. These results agree with previous findings by Baiden (2006)

\subsubsection{Coordinated working and relationship}

The clients, designers, contractors, system providers/manufacturers and installers emphasised that continuous and long-term working relationships would improve SC integration. A majority of the clients mentioned that the relationships should be continuously repeated among IBS players in order to improve SC integration. The designers expressed that having past working experience and established relationships are an added value in efficient design integration. Moreover, the designers expressed that, having professionally certified main contractors and system providers/manufacturers designers would better trust each other. This outcome was previously reported by Baiden (2006), who emphasised the importance of establishing SC relationships and noted the importance of continuous and long-term working relationships for improving SC integration.

\subsection{Working environments mean}

The importance of the work environment in successful supply chain integration was emphasised by the majority of contractors, system providers/manufacturers and installers. Overall, the results suggest that spatial interactions and no restrictions within the working environment enhance integration between IBS supply chains, especially those SC players that are more involved on the site. Although the lower tiers placed more emphasis on this factor, clients and other upper tier players should give higher consideration to the work environment. 
The importance of the work environment and spatial boundaries has been pointed out by Baiden (2006) as one of the important dimensions of integration.

\section{Conclusion}

Analysis of literature and data gained from interviews led to the following conclusion. Several factors associated with successful SC integration need to be considered by all players, from design to construction. Eight significant criteria under four means of success factors were identified based on multiple informant perspectives from different IBS SC. All IBS SC consistently ranked the category of "human and behavioural factors" as the most important, with "working culture and behaviour" and "competence and capability" as the top ranked factors in that category. Contractors also ranked "supply chain process and exchange flow" (specifically, "communication, information exchange and facilitate problem") and "supply chain structure and collaboration" (specifically, "procurement arrangements and responsibilities of supply chain" and "coordinated working relationships") as the highest priorities. However, even though different players sometimes identified different success factors, the differences and similarities in responses reflect the interconnectedness of the supply chain. The results emphasise the importance of making the right decisions towards improving integration in IBS project procurement environment. The main contribution especially for the decision makers who involve in project implementation will benefit this research by understanding the factors and are use these results to define better priorities and ensure continuous improvement as demanded in today's dynamic IBS construction environment.

\section{Acknowledgement}

The authors would like to thank the Construction Industry Development Board (CIDB) and UniversitiTeknologi MARA for providing the opportunity to participate in this conference.

\section{References}

Abd Shukor, A. S., Mohammad, M. F., Mahbub, R., Ismail, F. \& Halil, F. (2009). The implementation of supply chain management in the Malaysian construction industry. Paper presented at the International Conference on Construction and Real Estate Management (ICCREAM 2009), Beijing, China.

Baiden, B. K. (2006). Framework for the integration of the project delivery team. (Doctor of Philosophy), Loughborough University, Loughborough.

Briscoe, G. H. \& Dainty, Andrew R. J. (2005). Construction supply chain integration: an elusive goal? Supply Chain Management: An International Journal, 10(4), 319-326. 
Abd Shukor, A.S., et.al. / Asian Journal of Quality of Life, AjQoL,1(3), Sep / Oct 2016 (p.19-28)

CREAM (2009). 1st CIDB/CREAM IBS Roundtable Workshop (IRW01). Kuala Lumpur.

Handfield, R. B. \& Nichols, E. L. (1999). Introduction to supply chain management. Englewood Cliffs: Prentice-Hall.

Helou, M. M. \& Caddy, I. N. (2006). Definition problems and a general systems theory perspective in supply chain management. Problems and Perspectives in Management, 4(4), 77-83

Isnin, Z., Ramli, R., Hashim, A. Z \& Irwan, M. A. (2012). Are House Alterations Sustainable? Journal of Asian Behavioural Studies, 2(5), 13.

Jabar, I. L., Ismail, F., Aziz, N. M., Janipha, N. A. I. (2013). Construction manager's competency in managing the construction process of IBS project. Procedia Social and Behavioral Sciences, 105(2013), 85-93doi: 10.1016/j.sbspro.2013.11.010

Jorgensen, B. \& Emmitt, S. (2009). Investigating the integration of design and construction from a "lean " perspective Construction Innovation, 9(2), 225-240. doi: 10.1108/14714170910950849

Mohammad, M. F., Abd Shukor, A. S., Mahbub, R. \& Halil, F. (2014). Challenges in the Integration of Supply Chains in IBS Project Environment in Malaysia. Procedia Social and Behavioral Sciences,153 (2014), 44-54. doi: 10.1016/j.sbspro.2014.10.03

Shamsudin. S. M., Zakaria, R. \& Mohamed S. F. (2013). Economic Attributes in Industrialised Building System in Malaysia, Procedia - Social and Behavioural Sciences, 105(2013), 75-84. 